\title{
Colorectal Cancer Video for the Deaf Community: A Randomized Control Trial
}

\author{
Salma Shabaik • Sheila F. LaHousse $\cdot$ Patricia Branz • \\ Visha Gandhi • Amir M. Khan • Georgia Robins Sadler
}

Received: 5 March 2010 /Accepted: 5 March 2010/Published online: 15 April 2010

(C) The Author(s) 2010. This article is published with open access at Springerlink.com

\begin{abstract}
The Deaf community experiences multiple barriers to accessing cancer information. Deaf participants $(n=144)$ were randomly assigned to view a colorectal cancer education video or another program in American Sign Language. They completed surveys pre- and post-intervention and at 2 months post-intervention. By using a crossover model, control group participants were offered the option of seeing the intervention video. The experimental group gained and retained significantly more colorectal cancer knowledge than the control group, and the control group demonstrated the greatest knowledge gain after crossing into the experimental arm. This video effectively informed the Deaf community about colorectal cancer.
\end{abstract}

Keywords ASL · Cancer prevention · Colorectal cancer. Deaf $\cdot$ Education

S. Shabaik · S. F. LaHousse • P. Branz • V. Gandhi • A. M. Khan •

G. R. Sadler $(\bowtie)$

Moores UCSD Cancer Center,

3855 Health Sciences Drive no. 0850,

La Jolla, CA 92093-0850, USA

e-mail: gsadler@ucsd.edu

G. R. Sadler

Department of Surgery, UCSD School of Medicine,

La Jolla, CA, USA

S. Shabaik

UCSD School of Medicine,

La Jolla, CA, USA

S. F. LaHousse • G. R. Sadler

Department of Psychology, Michigan State University,

East Lansing, MI, USA

G. R. Sadler

SDSU/UCSD Joint Doctoral Program in Clinical Psychology,

San Diego, CA, USA

\section{Introduction}

It is estimated that 550,000 to one million residents of the United States and Canada self-identify as members of the Deaf community [1, 2, 31]. Through their use of American Sign Language (ASL) as their preferred or sole mode of communication and their affiliation with people who share their language and culture, they are considered to be members of the Deaf community [29-30]. The capital "D" denotes this community affiliation, in contrast to deaf with a lower case "d," which is an audiologic term.

ASL, with its unique grammar and syntax, uses hand gestures, facial expressions, and body movements, that are executed and understood within a rich spatial fabric to create pictures that transmit information [3]. Being a picture based language, ASL does not directly translate into written or spoken English [3, 4] and can only be expressed visually (e.g., in person, via video, or other mediums that provide for a visual field).

Within the Deaf community, English is generally learned as a second language without benefit of aural reinforcement, if it is learned at all. This leads to lower literacy rates, reported to be at the third to fourth grade level $[5,6,32,33]$, and creates language barriers. Both outcomes impede the Deaf community's access to information and integration into the mainstream community's learning activities.

Exacerbating the Deaf community's difficulty accessing printed health information is the complexity of the health education materials themselves, which are generally written at or beyond the tenth grade level [7-9]. Direct communication between health care providers and patients is rendered lessthan-optimal because few providers are competent in Deaf culture and even fewer are proficient in ASL. These barriers to communication can lead to the increased risk of miscommunication, as well as aborted communication attempts, which, 
in turn, can contribute to the creation of significant disparities in the Deaf community's health knowledge and practice of health-promoting activities [8, 10-13].

Little research has been conducted on understanding the Deaf community's knowledge of colorectal cancer (CRC). Sadler, et al. found $52 \%$ of surveyed Deaf women reported no prior knowledge of CRC. A recent survey of deaf clients at Chicago's two largest Deaf-serving health care systems found that $60 \%$ of respondents aged 50 years and older reported that a doctor had ever talked to them about CRC screening and 69\% rated screening as highly important [14]. An on-going relationship with a doctor was shown to increase the odds of being screened. Those who reported seeing a doctor three to five times in the past year were more likely to report that a doctor had ever talked to them about CRC screening in comparison to those who had seen a doctor zero to two times [14]. The study also found comparable screening rates for those aged 50 or older $(51 \%$ had a sigmoidoscopy or colonoscopy) to the US screening rate of $48 \%$ for either test in 2002 [14, 15].

CRC was the third most common cancer and third leading cause of cancer mortality in the USA from 1999 to 2003 [16]. Recent trends reveal a decline in CRC incidence and mortality that is believed to be directly correlated with increased adherence to recommended screening guidelines. Research is needed to find ways to motivate more people to follow recommended screening guidelines to help further decrease incidence and mortality rates [16-23]. This randomized education trial tested the effectiveness of a CRC education program in ASL with open captioning and voice over that was specifically created for the Deaf community.

\section{Materials and Methods}

Hypothesis 1 Deaf adults who participate in the ASL CRC video intervention will demonstrate significant $(p \leq 0.05)$ increases in general cancer and CRC knowledge than adults who do not.

Hypothesis 2 Adults who are primed with the survey questions prior to viewing the video intervention will demonstrate a significant $(p \leq 0.05)$ increase in $\mathrm{CRC}$ knowledge and retention than those who are not.

\section{Study Design}

Colorectal Cancer: Take Action! is a 60-min, graphically enriched video created by this educational partnership. It features native ASL signers explaining how CRC develops, risk factors, screening guidelines, diagnostic and treatment methods, and the importance of clinical trials participation.
This UCSD Institutional Review Board-approved study invited Deaf adults from Southern California to "participate in a health education study." After finishing the consenting process in English and/or ASL, all participants completed a baseline survey (survey A) about socio-demographic information, general cancer, and colorectal cancer knowledge. Participants were then randomized to either the experimental arm and viewed the ASL CRC video or the control arm and received the National Cancer Institute's clinical trials PowerPoint education program: The Basics that was explained in ASL and English. Participants completed an immediate postintervention survey (survey B) and a 2-month follow-up survey (survey C) with the same general cancer and colorectal cancer questions as the baseline survey.

The experimental arm participants received a copy of the video to view and share with family members and friends. Since the Deaf community has limited access to cancer information in ASL, the control arm participants were offered the opportunity to cross over into the experimental arm after completing the 2-month follow-up survey as controls.

\section{Data Analysis}

All data were entered and analyzed using SPSS version 14.0 [26]. To test the study's hypotheses, general cancer and CRC knowledge scales were created based on the content of the educational video; thus, these scales have not been previously validated. The scales included seven general cancer items and $13 \mathrm{CRC}$ items that were summed to create two overall sum scores: "general cancer knowledge" and "colorectal cancer knowledge." A binary coding system ( $1=$ correct, 0 = incorrect) was used for all items. Overall continuous sum scores were created for both scales for each of the three surveys administered (i.e., survey A, B, and C). General and CRC knowledge sum scores were analyzed over time using repeated measures analysis of variance and $t$ tests $[24,25]$. Chi-square tests were used to compare sociodemographic and communication variables between the study arms. Multivariate models including socio-demographic independent variables were run and did not change the results, and therefore only simple repeated measures of group by general cancer knowledge and group by CRC knowledge over time are reported. It was anticipated that members of the control arm who crossed over into the experimental arm might have a baseline shift in an unforeseen way. Thus, data analysis was conducted in three ways: with original members of the experimental arm, with members of the combined experimental group including those who crossed over, and with the crossover members only. The results are reported for the combined experimental group, unless the analysis revealed a significant difference.

The power calculations planned a sample size $(n=140)$ that would allow for a follow-up loss of $20 \%$ while still 
being able to demonstrate statistical significance at a $p \leq 0.05$ level [27]. In reality, 144 Deaf adults participated at baseline (86 in the experimental group and 58 controls), and the 2-month retention rates were: $94.2 \%$ for the experimental arm (81/86), $86.2 \%$ for the control arm $(50 / 58)$, and $64 \%$ for the crossover arm $(32 / 50$; see Fig. 1). An intent-to-treat analysis [28] was used to account those lost-to-follow-up. Generally, this is a conservative approach, but in this study using an intentto-treat method to substitute survey $\mathrm{C}$ scores with survey B scores for those crossover participants lost to the 2month follow-up may have biased results in favor of Hypothesis 2. To evaluate this, separate analyses were run to compare results without scores for those who were lostto-follow-up (without intent-to-treat) and results with scores using intent-to-treat. Since there was no significant difference between the analyses, only intent-to-treat scores are reported.
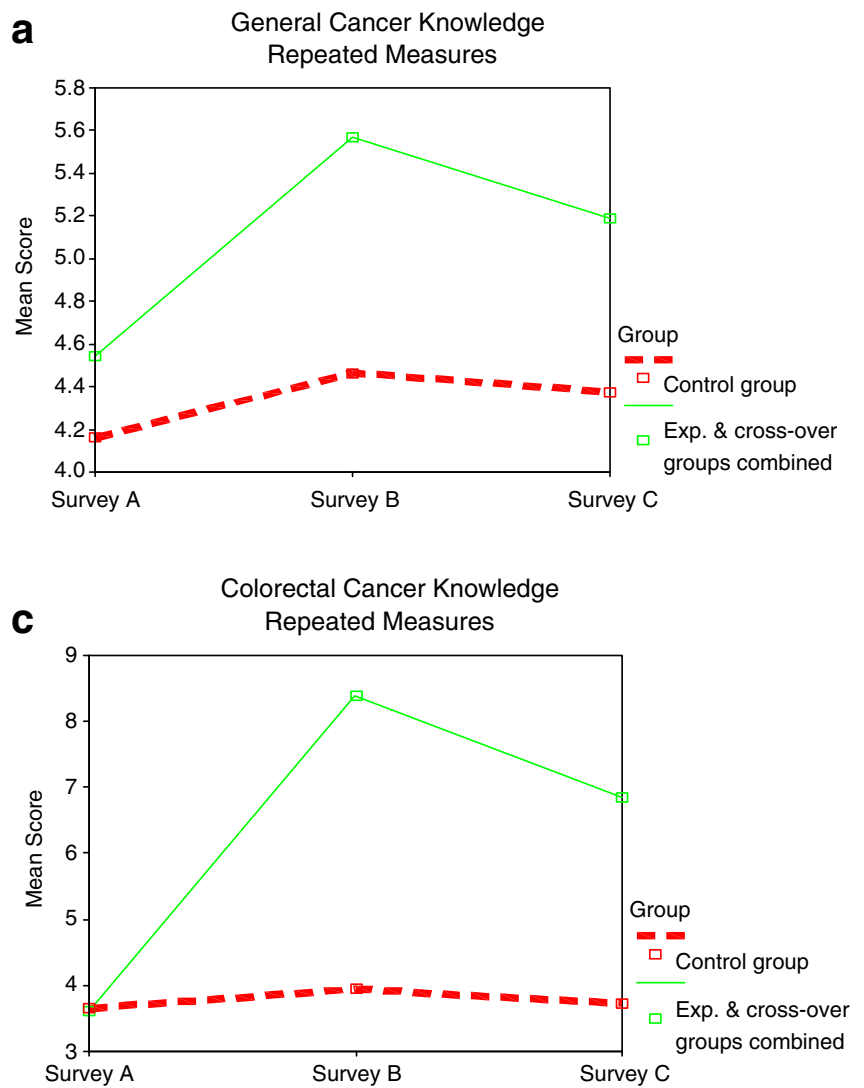

There were 83 women and 60 men in the study, with equivalent proportions in both study arms. One third of participants [33.6\% $(n=48)]$ reported having a college degree or beyond, with no significant difference between the study arms. Most $[84.4 \%(n=115)]$ defined themselves as either culturally Deaf or Deaf, $83.1 \%(n=118)$ preferred ASL as their primary method of communicating, 79.7\% $(n=114)$ reported writing notes back and forth when needing to communicate important information without an interpreter present, and $37.7 \%(n=52)$ reported usually communicating with their doctor through a sign language interpreter; no significant differences existed between the study arms for these variables. Experimental arm participants were on average 9.33 years older $(M=43.5, \mathrm{SD}=16.4, n=85)$ than those in the control arm $(M=34.2, \mathrm{SD}=13.6, n=58)$. Chi-square tests revealed differences of ethnicity and different ways of informing their doctor of a health problem between the two study arms $(p \leq 0.05)$.
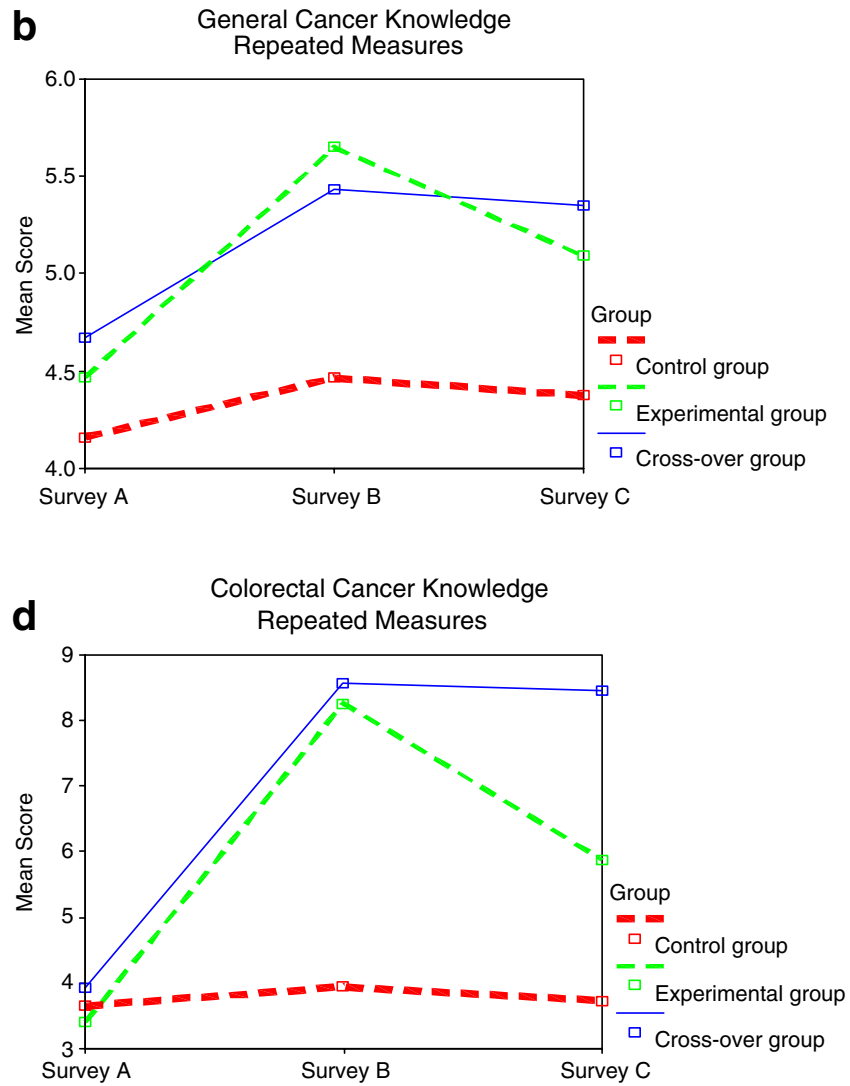

Note: General cancer knowledge scores range from 0-7 and colorectal cancer knowledge scores range from 0-13. Survey A was collected at baseline, Survey B immediately following the intervention, and Survey C at two-month follow-up.

Cross-over group's Survey A scores are their Survey C scores in the control group; this includes only those 50 subjects who opted to cross-over to the experimental arm.

Fig. 1 General cancer and colorectal cancer knowledge scores. Note: General cancer knowledge scores range from 0 to 7 and colorectal cancer knowledge scores range from 0 to 13 . Survey A was collected at baseline, survey B immediately following the intervention, and survey $\mathrm{C}$ at 2-month follow-up. Crossover group's survey A scores are their survey $\mathrm{C}$ scores in the control group; this includes only those 50 subjects who opted to cross over to the experimental arm 


\section{Results}

At baseline, there were no significant differences between the two groups' average scores for general cancer or CRC knowledge [control group $(M=4.2 ; M=3.7)$ and experimental group $(M=4.5 ; M=3.6)]$. However, there were differences in scores between kinds of knowledge at baseline, with both groups demonstrating a higher level of general cancer knowledge at baseline (experimental arm $=64.3 \%$ of questions answered correctly and control arm $=60.0 \%$ correct) then CRC knowledge (experimental $\mathrm{arm}=27.7 \%$ correct and control arm $=28.5 \%$ correct).

This may be a reflection of the growing cancer knowledge in the community from previous cancer programs or because $26.4 \%(38 / 144)$ of the sample participated in a previous cancer study.

Hypothesis 1 was supported. Post-intervention, Deaf individuals who had participated in the ASL CRC video intervention demonstrated significantly $(p \leq 0.05)$ higher scores when tested on CRC and general cancer knowledge than those who did not view that video. Specifically, the experimental arm demonstrated a significant increase in general cancer $\left(M_{\mathrm{A}}=4.5 ; M_{\mathrm{B}}=5.6 ; M_{\mathrm{C}}=5.2\right)$ and colorectal cancer $\left(M_{\mathrm{A}}=3.6 ; M_{\mathrm{B}}=8.4 ; M_{\mathrm{C}}=6.9\right)$ knowledge scores from point $\mathrm{A}$ to $\mathrm{B}(p \leq 0.05)$, with only a slight decrease (albeit statistically significant) in both average knowledge scores from survey B to C (Fig. 1a and c). Across the three points in time, the control arm demonstrated no significant change in general cancer $\left(M_{\mathrm{A}}=4.2 ; M_{\mathrm{B}}=4.5 ; M_{\mathrm{C}}=4.4\right)$ or CRC $\left(M_{\mathrm{A}}=3.7 ; M_{\mathrm{B}}=3.9 ; M_{\mathrm{C}}=3.7\right)$ knowledge scores.

In comparing the average mean change over time between arms, results showed that the experimental arm had a significant increase in general cancer (mean change $=1.04$, $p \leq 0.05$ ) and $\mathrm{CRC}$ (mean change $=4.8, p \leq 0.01$ ) knowledge from baseline (survey A) to immediately following intervention (survey B) in comparison to the control arm (general cancer mean change $=0.26$; CRC mean change $=0.23$ ).

While there was a statistically significant decrease in CRC knowledge in the experimental group from survey B to $\mathrm{C}$ (mean change $=-1.5$ ) compared to the control group, the increase in knowledge from survey $\mathrm{A}$ to $\mathrm{C}$ remained statistically significant. The experimental arm had a significant increase in average CRC knowledge scores (mean change $=3.2$ ) from $\mathrm{A}$ to $\mathrm{C}$ in comparison to the control arm (mean change $=0.09, p \leq 0.01$; Fig. $1 \mathrm{a}$ and $\mathrm{c}$ ). In contrast, for both groups, general cancer knowledge was unchanged from survey B to C and from A to C.

Hypothesis 2 was partially supported. Crossover participants did not have significantly higher scores from the original experimental participants for CRC knowledge immediately following the intervention (survey B), but crossover participants were significantly more likely to retain colorectal cancer knowledge at the 2-month follow-up (survey C).
The mean changes in CRC knowledge scores from survey A to $B$ and from survey $B$ to $C$ for the controls after crossover did not differ significantly from the experimental group. From survey $\mathrm{A}$ to $\mathrm{C}$, the mean change in colorectal cancer knowledge scores for the crossover arm (mean change $=4.4$ ) were significantly greater than the mean change of the original experimental arm (mean change $=2.4$; Fig. 1d), thus illustrating a greater overall gain of knowledge from baseline to 2-month follow-up for the crossover group.

In fact, the original experimental participants were significantly less likely to retain their CRC knowledge from immediately post-intervention (survey B; $M_{\mathrm{B}}=8.3$ ) to 2-month follow-up (survey $\mathrm{C} ; M_{\mathrm{C}}=5.9, p \leq 0.05$ ) in comparison to the crossover arm $\left(M_{\mathrm{B}}=8.6 ; M_{\mathrm{C}}=8.5\right)$. The mean change in general cancer knowledge scores over time (from surveys $\mathrm{A}$ to $\mathrm{B}, \mathrm{B}$ to $\mathrm{C}$, and $\mathrm{A}$ to $\mathrm{C}$ ) for the crossover group did not differ significantly from the original experimental arm (Fig. 1b).

\section{Discussion}

These data demonstrate that the ASL CRC video is a viable health education tool for the Deaf community. Participants gained and retained the desired knowledge points. One possible interpretation of the better outcomes for the crossover group is that the greater retention of the video's key information points might be enhanced by giving all future viewers a set of pre-intervention questions to review (vs. learning objectives) to help them focus on the essential points of information before showing them the video. However, further research is needed since it is also possible that the participants who chose the crossover option could have been more interested in the project's topic and more focused on retaining the information presented.

While the knowledge increase was significant, the retention was not the ideal of $100 \%$. However, this gain was accomplished with only a single viewing. Future research should also examine whether being exposed to the educational intervention more than once increases immediate and longer-term knowledge gain and retention. Future studies should also include larger sample sizes to account for the significant study attrition that occurs with a crossover design that begs an additional time commitment of participants. While this study demonstrated the video program's capacity to increase CRC knowledge, a subsequent study is warranted to determine if this video can also promote an increased uptake of screening among those individuals determined to be at higher-than-average risk of CRC.

The results also suggest that a stronger intervention impact occurred for CRC than general cancer knowledge, the probable result of the educational intervention's specific focus on CRC, with general cancer as a secondary focus. 
Finally, the relatively high baseline scores for general cancer knowledge may be a direct reflection of the growing impact this multi-year program is having on Southern California's Deaf community. Malcolm Gladwell has described the occurrence of such watershed phenomena, thereby providing a theoretical framework to support the outcome of such information diffusion [34].

\section{Conclusion}

These results cumulatively provide support that the ASL-based CRC education intervention did increase knowledge. This suggests that a video-based intervention is an effective educational tool for reaching the Deaf community with cancer information.

Acknowledgments The authors acknowledge the following sources of support for this study: The authors acknowledge the following sources of support for this study: NIH 5P30CA023100; NIH U56CA92079/ U56CA92081 and 1U54CA132379/1U54CA132384; NIH/NCMHD 5P60MD000220; NIH 5R25CA65745; NIH R25CA101317; NIH R25CA108731; UCSD Academic Senate Grant; The Susan G. Komen Breast Cancer Foundation, San Diego Affiliate Grant Award; Alliance Healthcare Foundation 99-99; and California Endowment. This project was created by an educational partnership consisting of: Moores UCSD Cancer Center, Deaf Community Services of San Diego Inc., Bovee Productions, the National Association for the Deaf, and Gallaudet University. The authors wish to also thank the following organizations that made the participant recruitment process possible: Gallaudet University; Deaf Community Services of San Diego, Inc. (DCS) in San Diego, CA, USA; Center on Deafness Inland Empire (CODIE) in Riverside, CA, USA; Orange County Deaf Equal Access Foundation (OC DEAF) in Cypress (Orange County, CA, USA); Deaf program with Disability Support Programs and Services at San Diego Mesa College in San Diego, CA, USA; and National Center on Deafness at California State University Northridge in Northridge, CA, USA.

Open Access This article is distributed under the terms of the Creative Commons Attribution Noncommercial License which permits any noncommercial use, distribution, and reproduction in any medium, provided the original author(s) and source are credited.

\section{References}

1. Mitchell RE et al (2006) How many people use ASL in the United States? Why estimates need updating. Sign Lang Stud 6(3):306-335

2. Mitchell RE (2005) How many deaf people are there in the United States? Estimates from the survey of income and program participation. J Deaf Stud Deaf Educ 11(1):112-119

3. Stokoe WC (1965) A dictionary of American sign language on linguistic principles. Lintok Press, Silver Spring, MD

4. Valli C, Lucas C (1992) Linguistics of American sign language: a resource text for ASL users. Gallaudet University Press, Washington, DC

5. Allen TE (1986) Patterns of academic achievement among hearing impaired students: 1974 and 1983. In: Schildroth AN, Kouchmer MA (eds) Deaf children in America. College-Hill Press, Boston, MA, pp 161-206
6. Holt JA (1993) Stanford Achievement Test - 8th edition: reading comprehension subgroup results. Am Ann Deaf Ref 138:172-175

7. Berland GK et al (2001) Health information on the Internet: accessibility, quality, and readability in English and Spanish. Jama 285(20):2612-2621

8. Institute of Medicine of the National Academics (IOM). Health Literacy: A Prescription to End Confusion. 2004 [cited 2007 November 1]; Available from: http://www.iom.edu/?id=19750

9. Perocchia RS et al (2005) Raising awareness of on-line cancer information: helping providers empower patients. J Health Commun 10(Suppl 1):157-172

10. Folkins A et al (2005) Improving the deaf community's access to prostate and testicular cancer information: a survey study. BMC Public Health 5(1):63

11. Sadler GR et al (2001) Bringing breast cancer education to deaf women. J Cancer Educ 16(4):225-228

12. Sadler GR et al (2001) Bringing health care information to the deaf community. J Cancer Educ 16(2):105-108

13. Steinberg AG et al (2006) Health care system accessibility. Experiences and perceptions of deaf people. J Gen Intern Med 21 (3):260-266

14. Orsi JM et al (2007) Cancer screening knowledge, attitudes, and behaviors among culturally deaf adults: implications for informed decision making. Cancer Detect Prev 31(6):474-479

15. Centers for Disease Control and Prevention (CDC). Behavioral Risk Factor Surveillance System (BRFSS) Prevalence Data: Colorectal Cancer Screening - 2002. 2007 [cited; Available from: http://apps.nccd.cdc.gov/BRFSS/list.asp?cat $=$ CC\&yr=2002\&qkey= $4425 \&$ state $=$ All

16. American Cancer Society (ACS). Cancer Facts \& Figures, 2007. 2007 [cited 2007 September 21]; Available from: http://www. cancer.org/downloads/STT/CAFF2007PWSecured.pdf

17. Agency for Healthcare Research and Quality (AHRQ). The Guide to Clinical Preventive Services: Recommendations of the U.S. Preventive Services Task Force, U.S. Department of Health and Human Services. 2006 [cited 2007 September 21]; Available from: http://www.ahrq.gov/clinic/cps3dix.htm\#cancer

18. Bond JH (2006) The case for direct colonoscopy screening for colorectal cancer. Am J Gastroenterol 101(2):263-265

19. Espey DK et al (2007) Annual report to the nation on the status of cancer, 1975-2004, featuring cancer in American Indians and Alaska Natives. Cancer 110(10):2119-2152

20. Meissner HI et al (2006) Patterns of colorectal cancer screening uptake among men and women in the United States. Cancer Epidemiol Biomarkers Prev 15(2):389-394

21. United States Preventive Services Task Force Agency for Healthcare Research and Quality. Screening for Colorectal Cancer: Recommendations and Rationale. 2002 [cited 2007 November 1]; Available from: http://www.ahrq.gov/clinic/3rduspstf/colorectal/colorr.htm

22. Walsh JM et al (2005) Promoting use of colorectal cancer screening tests. Can we change physician behavior? J Gen Intern Med 20(12):1097-1101

23. Levin B et al (2008) Screening and surveillance for the early detection of colorectal cancer and adenomatous polyps: a joint guideline from the American Cancer Society, the US MultiSociety Task Force on Colorectal Cancer, and the American College of Radiology. CA Cancer J Clin 58(3):130-160

24. Cohen J et al (2003) Applied multiple regression/correlation analysis for the behavioral sciences, 3rd edn. Lawrence Erlbaum Associates, Hillsdale, NJ

25. Kleinbaum DG et al (2007) Applied regression analysis and multivariable methods, 4th edn. Duxbury Press, Pacific Grove, CA

26. SPSS for Windows Version 14.0.0. 2005. SPSS Inc., Chicago

27. Lenth RV (2001) Some practical guidelines for effective sample size determination. The American Statistician 55:187-193 
28. Newell DJ (1992) Intention-to-treat analysis: implications for quantitative and qualitative research. Int J Epidemiol 21(5):837841

29. Woodward J (1982) How you gonna get to heaven if you can't talk with Jesus: on depathologizing deafness. T. J. Publishers, Inc., Silver Spring, MD

30. Padden C, Humphries T (1988) Deaf in America: voices from a culture book. Harvard University Press, Allentown, PA

31. DawnSignPress. The Deaf-World; 2003. Available from URL: http://www.dawnsignpress.com/journey/index.html [accessed 2008 January 2]
32. Allen TE (1994) A comprehensive evaluation of the postsecondary educational opportunities for students who are deaf or hard of hearing. Paper submitted to Pelavin Research Institute as part of the project and funded by the U.S. Office of Special Education and Rehabilitative Services

33. Bat-Chava Y, Rosen RB, Sausa A, Meza C, Shockett S, Deignan E (1999) An evaluation of a college preparatory and readiness program for deaf students. J Rehabil 65:51-59

34. Gladwell M (2000) The tipping point: how little things can make a big difference, 1st edn. Little, Brown, and Company, Boston 